\title{
Letters
}

All letters are subject to editing and may be shortened. General letters can be sent to bjgpdisclarcgp.org.uk (please include your postal address for publication), and letters responding directly to BJGP articles can be submitted online via eLetters. We regret we cannot notify authors regarding publication. For submission instructions visit: bjgp.org/letters

A larger than normal number of letters have been received this month, so it has only been possible to select a few for publication.

\section{Triage of patients with COVID-19}

Many medical bodies have issued guidance over the 4 months since COVID-19 was identified. By my reckoning there are at least 12 National Institute for Health and Care Excellence COVID-19 rapid guidelines and at least 20 other sets of national guidance from clinical leaders in many fields. The Royal College of General Practitioners and British Medical Association among others have resource hubs, and local systems are doing their best to interpret this information and apply it with more guidelines and system changes.

As each new guidance hits my inbox I feel a mixture of slight irritation and a spark of interest to see if there is a nugget of information that I can apply in my day-today decision making.

Some things however have occurred to me:

1. We can't know that much about an illness that is only 4 months old and it is amazing that it is possible to write so much guidance about it.

2. I get a sense that people are sometimes trying to fill the knowledge gap too soon lthe Roth score being used in triage of patients with COVID-19 being an example of this). We should be prepared to say sometimes, 'I'm afraid that you are on your own here with your clinical judgement because we really don't have an evidence-based approach yet.

3. In GP Out of Hours $(\mathrm{OOH})$ work in particular it currently feels like there is a lot of pressure placed on us to triage away from face-to-face consultations. One is aware that you are potentially putting a colleague's life at risk and systems are being put in place to put bureaucratic hoops in the way of seeing patients face to face. Patients also are strongly resisting face-to-face assessments. The 'Stay at home' mantra is very hard to overcome.

4. Yet telephone or video triage for COVID-
19 patients has little or no evidence base, and I have certainly been surprised by finding a number of frailer patients who don't look or sound too bad, with oxygen saturations well less than $90 \%$ or other observations consistent with sepsis.

5. In general practice we have a tendency to discriminate against the inconvenient (for example, $\mathrm{OOH}$ work) and the unpleasant. Of course we need to minimise the risks to clinicians in assessing patients with possible symptoms of COVID-19 but we need to acknowledge our own discomfort when making triage decisions on the telephone or by video link, and also the lack of an evidence base for this work currently.

6. If in doubt, recognising the challenges of limited supplies of personal protective equipment (PPE), isolation rooms, and challenges of cleaning rooms and equipment, we need to have systems in place where people can be seen if they need to be. If nothing else, to try to reduce the number of anxious hyperventilating patients that I am currently seeing from ending up inappropriately in a hospital COVID-19 assessment unit.

7. Excessive pressure to avoid face-to-face consultations might risk an unnecessary complaint or an adverse medical outcome.

Alex Manning,

Burwell Surgery, Newmarket Road,

Burwell, Cambridge.

Email: alex.manninglanhs.net

DOI: https://doi.org/10.3399/bjgp20X710825

\section{COVID-19 cumulative mortality rates for frontline healthcare staff in England}

We aimed to determine the mortality rates of the main groups of frontline healthcare providers (HCPs) with COVID-19.

Data on deaths of frontline HCPs in England with COVID-19 were collected through searches of news media reports. ${ }^{1,2}$ HCPs were categorised as doctor, nurse, or other lincluding ambulance, scientific, technical, and support staff, and direct care staff in general practices). We used December 2019 data for the corresponding numbers of registered HCPs in each occupation category,3,4 and calculated crude cumulative weekly mortality rates per 1000 for each category, as well as for the general population. ${ }^{5,6}$ We were unable to stratify analyses by ethnicity as the ethnic composition of each occupation category was not available.

Of the 147 frontline HCPs in England who died from COVID-19 between 25 March 2020 and 13 May 2020 for whom we had data, doctors accounted for $19.1 \%$ ( $n=28$, including $10 \mathrm{GPs}$ ), nurses $42.9 \%$ $(n=63)$, and other HCPs $38.1 \%(n=56)$. Doctors experienced the earliest reported deaths among HCPs, but the cumulative mortality rate for nurses was comparable with doctors by the week of 18 April. The cumulative mortality rates were 0.15 per 1000 doctors, 0.17 per 1000 nurses, and 0.10 per 1000 other HCPs, compared with 0.74 per 1000 people in the English general population. From the available information, 35 out of $97(36.1 \%)$ were aged 60 years or more, 68 out of 147 (46.3\%) were male, and 97 out of 128 (75.8\%) were from nonwhite ethnic backgrounds. The mean age of white HCPs was 59.2 years compared with 54.6 years for non-white HCPs.

Although frontline HCPs may have had greater exposure than the general population, the relatively lower mortality rates in the HCP groups may be due to a number of factors: greater access to professional protection equipment (PPE), lower rates of comorbidity, and fewer very older people than the general population, although levels of non-white ethnicity are higher in HCPs. COVID-19 mortality has been under-reported, but over one-third of the doctors in our list were GPs despite rises in remote consulting. The increased risks to community-based HCPs should not be overlooked.

Louis S Levene,

Department of Health Sciences, George Davies Centre, University of Leicester, Leicester; Diabetes Research Centre, Leicester General Hospital, University of Leicester, Leicester.

Email: Isl7aleicester.ac.uk 
Briana Coles,

Leicester Real World Evidence Unit, Diabetes Research Centre, Leicester General Hospital, University of Leicester, Leicester.

Melanie J Davies,

Diabetes Research Centre, Leicester General Hospital, University of Leicester. Leicester.

Wasim Hanif,

Queen Elizabeth Hospital, Edgbaston, Birmingham.

Francesco Zaccardi,

Leicester Real World Evidence Unit, Diabetes Research Centre, Leicester General Hospital, University of Leicester, Leicester.

Kamlesh Khunti,

Leicester Real World Evidence Unit, Diabetes Research Centre, Leicester General Hospital, University of Leicester. Leicester.

\section{Funding}

None.

\section{Ethical approval}

All data used were within the public sector and no ethical approval was required. Summary data are presented so no individuals are identifiable.

\section{Acknowledgements}

We acknowledge the support from the National Institute for Health Research (NIHR) Applied Research Collaboration East Midlands (ARC EM) and the University of Leicester Real World Evidence Unit. The views expressed are those of the authors.

\section{REFERENCES}

1. Lyons I, Penna D, Ward V, et al. These are the health workers who have died from coronavirus. Telegraph 2020; 20 May: https://www.telegraph. co.uk/news/0/nhs-workers-died-coronavirusfrontline-victims/ (accessed 10 Jun 2020)

2. Marsh S. Doctors, nurses, porters, volunteers: the UK health workers who have died from Covid- 19. Guardian 2020; 22 May: https://www.theguardian. com/world/2020/apr/16/doctors-nurses-portersvolunteers-the-uk-health-workers-who-havedied-from-covid-19 (accessed 10 Jun 2020).

3. NHS Digital. NHS workforce statistics December 2019. 2020. https://digital.nhs.uk/ data-and-information/publications/statistical/nhsworkforce-statistics/december-2019 laccessed 10 Jun 2020).
4. NHS Digital. General practice workforce 31 December 2019. 2020. https://digital.nhs.uk/ data-and-information/publications/statistical/ general-and-personal-medical-services/final-31december-2019 (accessed 10 Jun 2020).

5. NHS England. COVID-19 daily deaths. 2020. https://www.england.nhs.uk/statistics/statisticalwork-areas/covid-19-daily-deaths/ laccessed 10 Jun 2020)

6. Office for National Statistics. Subnational population projections for England: 2018-based. 2020. https://wmw.ons.gov. uk/peoplepopulationandcommunity/ populationandmigration/ populationprojections/bulletins/ subnationalpopulationprojectionsforengland/ 2018based laccessed 10 Jun 2020).

\section{Reduction in face-to- face GP consultations}

The COVID-19 pandemic has produced the biggest change in consultation patterns in British general practice for at least 200 years. The St Leonard's Practice in Exeter has for years recorded and analysed consultation data. ${ }^{1,2}$

After NHS guidance to reduce face-toface consultations, there was an immediate reduction. During March the number of face-to-face consultations fell from a mean of 52.1 (95\% confidence interval $[\mathrm{Cl}]=35.2$ to 67.1) per weekday in the 2 weeks before the change to a mean of $3.9195 \% \mathrm{Cl}=1.9$ to 5.9) for the second half of the month, that is, a $92.5 \%$ decrease $(P<0.001)$. The number of telephone consultations increased on average by $85.6 \%(P=0.0013)$ in the same time period, with a mean of 50.1 (95\% $\mathrm{Cl}=40.7$ to 57.5 ) per weekday in the first two weeks of March to a mean of $93.0195 \%$ $\mathrm{Cl}=70.5$ to 115.5 ) in the final weeks of the month.

We have produced a table showing appointments per 100 patients by patient age group, before and after (Table 1).
The practice also began using eConsults and the use of these by age groups is shown on the right-hand side of the table with proportionately more use of eConsults by patients aged $<65$.

Patients in the lowest two national deciles of the Index of Multiple Deprivation numbered 1324 (14\% of the list) and their consultations formed $17.2 \%$ of all consultations in the first half of the month and $16.8 \%$ afterwards, showing no significant difference ( $P=0.8)$.

This big reduction in face-to-face consultations as a result of the pandemic will have occurred in many other practices. We hope this quantification will assist future planning.

\section{Denis Pereira Gray,}

St Leonard's Research Practice, Exeter. Email: denis.pereiragraylabtinternet.com

Kate Sidaway-Lee,

St Leonard's Research Practice, Exeter.

Alex Harding,

St Leonard's Research Practice, Exeter.

Philip Evans,

St Leonard's Research Practice, Exeter.

\section{REFERENCES}

1. Pereira Gray DJ. The key to personal care. J R Coll Gen Pract 1979; 29(208): 666-678.

2. Sidaway-Lee K, Pereira Gray D, Evans P. A method for measuring of continuity of care in day-today general practice: a quantitative analysis of appointment data. Br J Gen Pract 2019; DOI: https://doi.org/10.3399/bjgp19X701813.
Table 1. Consultations per 100 registered patient by patient age group and consultation type before and after instigation of lockdown

Consultations per 100 registered patients

\begin{tabular}{lllllll}
\hline & \multicolumn{5}{c}{ Consultations per $\mathbf{1 0 0}$ registered patients } \\
\cline { 2 - 7 } $\begin{array}{l}\text { Age group, } \\
\text { years }\end{array}$ & Face-to-face & Telephone & eConsult & Face-to-face & Telephone & eConsult \\
\hline $0-19$ & 4.11 & 4.66 & 0 & 0.49 & 6.53 & 1.65 \\
\hline $20-64$ & 2.28 & 2.07 & 0 & 0.39 & 12.01 & 3.66 \\
\hline$\geq 65$ & 9.35 & 6.49 & 0 & 0.97 & 18.51 & 1.23 \\
\hline
\end{tabular}

